\title{
The replacement correction factors for cylindrical chambers in high-energy photon beams
}

\author{
L L W Wang and D W O Rogers \\ Ottawa Carleton Institute of Physics, Carleton University Campus, Ottawa, K1S 5B6, Canada
}

Received 25 November 2008, in final form 13 January 2009

Published 19 February 2009

Online at stacks.iop.org/PMB/54/1609

\begin{abstract}
The values of the replacement correction factors ( $P_{\text {repl }}$, or in the IAEA's notation $\left.p_{\text {dis }} p_{\text {cav }}\right)$ used for cylindrical chambers in high-energy photon beams represent one of the most significant differences between the AAPM and the IAEA dosimetry protocols. In a previous study (Wang L L W and Rogers D W O 2008 Med. Phys. 35 1747-55), we found that the AAPM protocol adopted incorrect values of $P_{\text {repl }}$ for cylindrical chambers in photon beams. For a ${ }^{60} \mathrm{Co}$ beam, the calculated $P_{\text {repl }}$ value is $0.5 \%$ higher than the AAPM value and about $1 \%$ higher than the IAEA value. It was still not clear why the IAEA values, which are based on measurements by Johansson et al, are incorrect. In this study, EGSnrc Monte Carlo simulation codes are used to simulate Johansson et al's experimental procedures for determining $P_{\text {repl }}$ values. The simulation results agree well with the measurements if the chamber responses versus depth are normalized at $d_{\max }$ as was apparently done in the experiments as it was believed that the chambers of different radii gave the same maximum reading at the respective $d_{\max }$. However, if the chamber responses are not normalized, then the simulated experimental results lead to a result which agrees well with the $P_{\text {repl }}$ values calculated by the standard Monte Carlo methods. This demonstrates that the normalization procedure used in the experiments is incorrect as is based on an incorrect assumption, and thus the interpretation of Johansson et al's experimental values as $P_{\text {repl }}\left(p_{\text {dis }}\right)$ in the IAEA TRS-398 Code of Practice is wrong. The values of $P_{\text {repl }}$ for cylindrical chambers of different radii in various high-energy photon beams are calculated and an empirical formula is given.
\end{abstract}

(Some figures in this article are in colour only in the electronic version)

\section{Introduction}

In ion chamber dosimetry the replacement correction factor ( $P_{\mathrm{repl}}=P_{\mathrm{gr}} P_{\mathrm{fl}}$, or in IAEA's notation $\left.p_{\text {dis }} p_{\text {cav }}\right)$ corrects for the changes of the shape and amplitude in the electron fluence spectrum due to the cavity of the ion chamber. In photon beams, since the fluence correction 
$P_{\mathrm{fl}}$ (or $p_{\text {cav }}$ ) is taken as unity, $P_{\text {repl }}$ is just the gradient correction $P_{\mathrm{gr}}\left(p_{\mathrm{dis}}\right)$. According to the IAEA, for cylindrical chambers in high-energy photon beams, the value of $P_{\mathrm{gr}}$ 'is one of the major contributions to the final uncertainty in $k_{Q}$ ' (IAEA 2001). The estimated uncertainty of the $P_{\mathrm{gr}}$ ratio entering into the $k_{Q}$ value is $0.5 \%$ (IAEA 2001). The values of $P_{\text {repl }}$ used in the two major current dosimetry protocols are also significantly different (Rogers 1996). For example, the AAPM's TG-51 (Almond et al 1999) and TG-21 (AAPM $1983)$ dosimetry protocols use a value of $P_{\text {repl }}=0.992(0.995)$ for a Farmer chamber in a ${ }^{60} \mathrm{Co}(18 \mathrm{MV})$ beam and the IAEA's TRS-398 Code of Practice (2001) uses a value of 0.988 (0.994) for the same quantities. This discrepancy is the most significant difference between the AAPM and the IAEA protocols for photon beam dosimetry. The AAPM's $P_{\text {repl }}$ values are based on the work of Cunningham and Sontag (1980) who derived $P_{\text {repl }}$ values from a combination of measurements and analytical calculations. It was pointed out in our previous study (Wang and Rogers 2008a) that the interpretation of Cunningham and Sontag's measured values as $P_{\text {repl }}$ in the TG-21 dosimetry protocol is not correct: the actual $P_{\text {repl }}$ value for a Farmer chamber in a ${ }^{60} \mathrm{Co}$ beam is $0.997,0.5 \%$ higher than that used in AAPM protocols and about $1 \%$ higher than that in the IAEA protocols. The values used in the IAEA Code of Practice are based on the experimental measurements of Johansson et al (1977). Andreo et al (1991) have tried to resolve this discrepancy between the AAPM and the IAEA protocols by using the ACCEPT/ITS (Halbleib 1988) Monte Carlo codes to calculate the response of cylindrical chambers in a ${ }^{60} \mathrm{Co}$ beam, but did not reach a definite conclusion, partly because the calculation's statistical uncertainty was too large.

Johansson et al (1977) measured $P_{\mathrm{gr}}\left(p_{\mathrm{dis}}\right)$ using two approaches. In one they measured the offset required with depth-dose curves measured using cylindrical chambers with different radii compared to that with a plane-parallel chamber. This established an offset of $0.6 r_{\text {cav }}$ which is used by both TG-51 and TRS-398 for measuring depth-dose curves. In a second approach they measured a displacement factor (DF) for depths greater than $d_{\max }$ from which the multiplicative correction factor $P_{\mathrm{gr}}=1-\mathrm{DF} r_{\mathrm{cav}}$ could be obtained. This was done by measuring photon beam depth-ionization curves with ion chambers with the same wall materials and diameters of 3,5 and $7 \mathrm{~mm}$. On the assumption that $P_{\mathrm{gr}} P_{\mathrm{fl}}=1$ at $d_{\max }$, they normalized the depth-ionization curves at $d_{\max }$ to effectively convert the measured ionizations to ionizations per unit mass of air in the chambers. From these curves they extracted the DF values. As we will show below, the normalization procedure is not correct and leads to incorrect values of DF and hence $P_{\text {gr }}\left(p_{\text {dis }}\right)$ as used in the IAEA's TRS-398. The EGSnrc (Kawrakow and Rogers 2000, Kawrakow 2000a) Monte Carlo simulation codes are used in this study to simulate the experimental procedures of Johansson et al (1977) for determining the displacement factors DF. The simulation results are compared to the measurements. The values of $P_{\text {repl }}$ for cylindrical chambers of different radii in various high-energy photon beams are calculated and an empirical formula for $P_{\text {repl }}$ values of cylindrical chambers in photon beams is given.

\section{Simulation of Johansson's experiments}

\subsection{The EGSnrc Monte Carlo simulation}

Johansson et al (1977) measured the depth-ionization curves in a PMMA phantom by using cylindrical chambers of different diameters $(3,5$ and $7 \mathrm{~mm}$ ) for photon beams ranging from ${ }^{60} \mathrm{Co}$ to $42 \mathrm{MV}$. The wall material for these chambers was also PMMA with a $50 \mu \mathrm{m}$ graphite internal coating. These depth-ionization curves were normalized at the depth of maximum ionization, $d_{\max }$. The ratios of the relative ionizations in different chambers 
were determined for depths larger than $d_{\max }$. The displacement factor (DF) was obtained by (Karl-Axel Johansson, 2008, private communication)

$$
\mathrm{DF}\left(\% \mathrm{~mm}^{-1}\right)=\frac{1-\left(J_{\mathrm{air}, 1}^{\prime} / J_{\mathrm{air}, 2}^{\prime}\right)}{r_{2}-r_{1}}
$$

where $J_{\text {air,1 }}^{\prime} / J_{\text {air,2 }}^{\prime}$ is the ratio of the relative ionization per unit mass of air for chambers 1 and 2 (hereafter referred to as the mass ionization), $r_{1}\left(r_{2}\right)$ is the radius of chamber 1 (chamber 2 ), assuming $r_{2}>r_{1}$. Basically, equation (1) tells us that the relative difference of the two chamber responses is caused by the difference in the chamber radius. In other words, the higher response for the larger chamber is due to the displacement of the phantom material by air in a cylindrical shell from $r_{1}$ to $r_{2}$. Since they did not know the absolute mass ionization for these cylindrical chambers, Johansson et al measured the relative ionization $J_{\text {air }}^{\prime}$ for the 5 and $7 \mathrm{~mm}$ diameter chambers normalized to the $3 \mathrm{~mm}$ chamber in a variety of photon beams of different energies. They found that the ratios of the two ionizations (i.e., $5 \mathrm{~mm}$ to $3 \mathrm{~mm}$, or $7 \mathrm{~mm}$ to $3 \mathrm{~mm}$ ) at $d_{\max }$ was independent of the photon beam quality. They then assumed that the fluence perturbation was negligible near $d_{\max }$ where there was an approximate charged particle equilibrium. In addition, the gradient correction was also negligible near $d_{\max }$. They concluded that the measured relative ionization represented the relation of the effective air mass between chambers and the mass ionization at $d_{\max }$ for different chambers must be the same. That means the mass ionizations in equation (1), $J_{\text {air, } 1}^{\prime}$ and $J_{\text {air, } 2}^{\prime}$, were actually normalized to the respective mass ionization at $d_{\max }$.

We simulated the experiments with the EGSnrc user-code Cavity (Kawrakow 2005) by modeling two cylindrical cavities of diameters 3 and $7 \mathrm{~mm}$ at various depths in a PMMA phantom of size $30 \times 30 \times 30 \mathrm{~cm}^{3}$, irradiated by a ${ }^{60} \mathrm{Co}$ beam and linac photon beams of energies from $4 \mathrm{MV}$ to $18 \mathrm{MV}$. To see if the graphite coating influences the calculated chamber response, we also modeled the air cavities with a cylindrical shell of graphite of thickness $50 \mu \mathrm{m}$ on the inner surface of the PMMA wall with the cavity centered at $10 \mathrm{~cm}$ depth in the PMMA phantom irradiated by the ${ }^{60} \mathrm{Co}$ beam. The results show that there is no significant difference at the $0.1 \%$ level. In other words, the graphite coating can be ignored in the simulation. The spectrum for the ${ }^{60} \mathrm{Co}$ beam is from Mora et al (1999). The spectra for the linac beams are from Monte Carlo simulations of a Varian linac (Sheikh-Bagheri and Rogers 2002). The ${ }^{60} \mathrm{Co}$ beam is a point source at $80 \mathrm{~cm}$ source-surface distance (SSD), the linac beams are point sources at $100 \mathrm{~cm} \mathrm{SSD}$, and all the field sizes are $10 \times 10 \mathrm{~cm}^{2}$. The cutoff energy for charged particles and photons are $521 \mathrm{keV}$ (10 keV kinetic energy) and $10 \mathrm{keV}$, respectively. The photon splitting variance reduction technique (Kawrakow 2005) is used and the splitting number varies from 20 to 100 depending on the radiation source quality. The number of histories used for the simulation is from 1 to 10 billion depending on the chamber cavity size and the photon splitting number used. The statistical uncertainty of the calculated cavity dose for the two modeled chambers varies from $0.06 \%$ to $0.1 \%$. Since the $W / e$ factor (the average energy required to produce an ion pair in dry air) is believed to be constant, the chamber cavity dose is proportional to the mass ionization of the chamber, i.e. $D_{\mathrm{air}, 1} / D_{\mathrm{air}, 2}=J_{\mathrm{air}, 1} / J_{\mathrm{air}, 2}$, where $D_{\mathrm{air}, i}$ and $J_{\mathrm{air}, i}$ are the absolute dose and the absolute mass ionization in the cavity of chamber $i$, respectively. For this reason, the terms 'cavity dose' and 'mass-ionization' or 'chamber response' will be used interchangeably in this study. Figure 1 shows the calculated cavity dose near $d_{\max }$ for the two modeled chambers in the ${ }^{60} \mathrm{Co}$ beam, together with the calculated depth-ionization curve calculated from the depth-dose curve corrected for the PMMA/air stopping power ratio (with $\Delta=10 \mathrm{keV}$ ). The calculated cavity dose is the absolute dose in the cavity per unit incident photon fluence and the depth-ionization curve is the absolute dose to air in a perturbation-free cavity per unit incident photon fluence. 


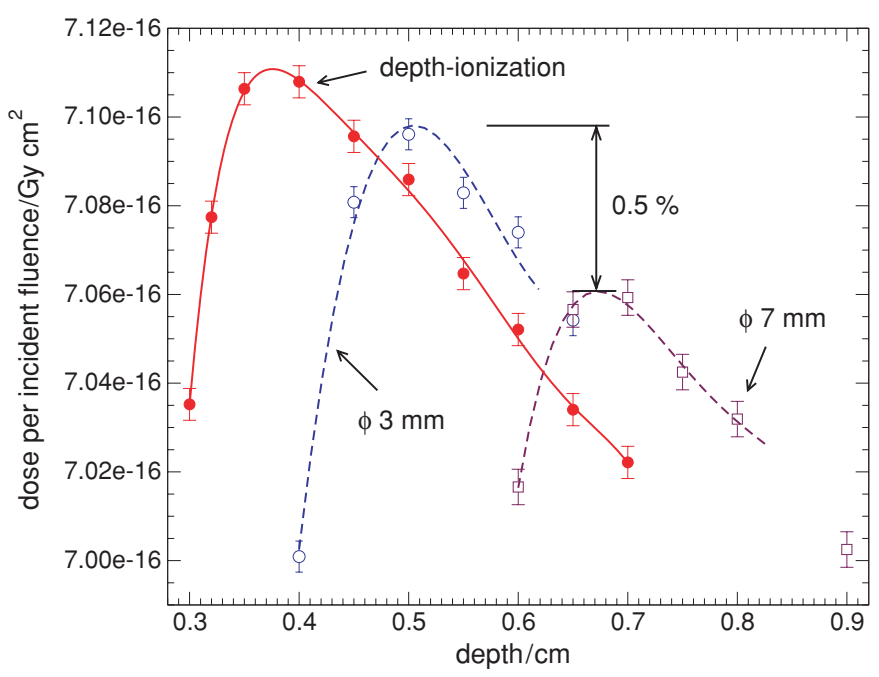

Figure 1. Calculated chamber cavity dose (open symbols), which is proportional to the massionization or chamber response, near depth of maximum dose in a PMMA phantom irradiated by a ${ }^{60} \mathrm{Co}$ beam of $80 \mathrm{~cm} \mathrm{SSD}$ and $10 \times 10 \mathrm{~cm}^{2}$ field size. Two chambers of diameters $3 \mathrm{~mm}$ and $7 \mathrm{~mm}$ are modeled. The solid circles are the calculated depth-ionization curve based on a depthdose curve corrected by the PMMA/air stopping power ratio with $\Delta=10 \mathrm{keV}$. Lines are the polynomial fits to the symbols, respectively.

The only purpose of the polynomial fitting for the calculated points is to find $d_{\max }$ and the maximum cavity dose (or maximum mass-ionization or response). Contrary to Johansson et al's belief, the mass ionization at $d_{\max }$ for the $7 \mathrm{~mm}$ chamber is not the same as that of the $3 \mathrm{~mm}$ chamber, and both of them are lower than the maximum value on the depth-dose curve. This issue will be discussed further in the next section. For now, our purpose is to repeat the experimental procedures by Monte Carlo simulation, so the obtained maximum mass ionizations are subsequently used in the normalization of the relative mass ionizations at all depths, as was done in the experiments. The same procedure (i.e., calculating the depthionization curve, finding the maximum mass ionization, and normalizing at $d_{\text {max }}$ ) is repeated for the $4 \mathrm{MV}$ and $18 \mathrm{MV}$ photon beams. Using equation (1), the displacement factors are calculated for the three photon beams at a variety of depths in the phantom. At most of these depths, the electron contamination is negligible.

\subsection{The simulation results and discussions}

The calculated displacement factors from the simulation of Johansson's experiments are compared to the measurements in figure 2 as a function of depth in phantom. There is excellent agreement for the ${ }^{60} \mathrm{Co}$ beam; and good agreement is also obtained for the $4 \mathrm{MV}$ and the $18 \mathrm{MV}$ beams, although they are compared to the measured values for a $5 \mathrm{MV}$ and a $16 \mathrm{MV}$ beam, respectively. We do not have photon spectral data for a $5 \mathrm{MV}$ or a $16 \mathrm{MV}$ beam and, more importantly, those linacs were manufactured about 30 years ago; thus, even with the same accelerating voltage, the actual photon beam quality may not be the same as those from a modern linac. For the $4 \mathrm{MV}$ beam, the lower value of the displacement factor at $5 \mathrm{~cm}$ is likely due to the statistics. For the $18 \mathrm{MV}$ beam, the lower values of the displacement factor at 4 and $5 \mathrm{~cm}$ are because of the lack of charged particle equilibrium at these depths for this high-energy beam. Note that the experimental values for the ${ }^{60} \mathrm{Co}$ beam vary significantly 


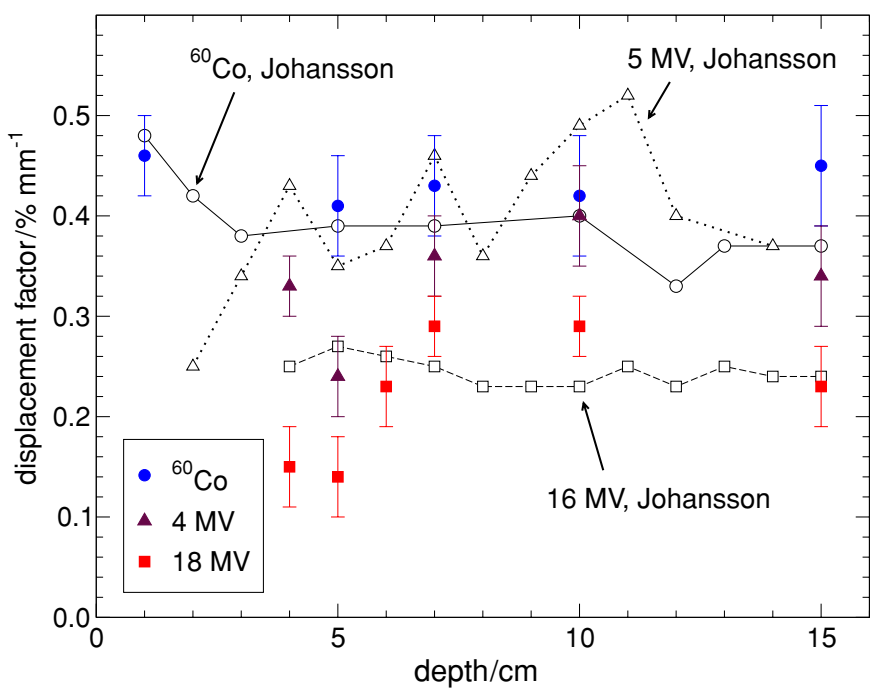

Figure 2. Measured displacement factors (open symbols) for a ${ }^{60} \mathrm{Co}$, a $5 \mathrm{MV}$ and a $16 \mathrm{MV}$ photon beam (data from Johansson et al (1977)) as compared to the calculated values (solid symbols) for a ${ }^{60} \mathrm{Co}$, a $4 \mathrm{MV}$ and an $18 \mathrm{MV}$ photon beam as a function of depth in a PMMA phantom. The calculated values are from the simulation of the two chambers of diameters $3 \mathrm{~mm}$ and $7 \mathrm{~mm}$.

from 0.33 to 0.48 ; and the measured values of the displacement factor for the $5 \mathrm{MV}$ beam are even more dispersed as a function of depth. This indicates that the method itself is not reliable in determining the displacement factor. If one takes the variation of the displacement factor DF versus the variation of the mass ionization ratio $R=J_{\text {air }, 1}^{\prime} / J_{\text {air, } 2}^{\prime}$ in equation (1), one obtains

$$
\frac{\delta \mathrm{DF}}{\mathrm{DF}}=\frac{-R}{1-R} \frac{\delta R}{R} .
$$

As $R$ is very close to 1 , equation (2) tells us that the relative uncertainty of the displacement factor could be very large. In fact, for the two chambers of diameters $3 \mathrm{~mm}$ and $7 \mathrm{~mm}$, the ratio $R$ is about 0.99 at all depths for a ${ }^{60} \mathrm{Co}$ beam, and even closer to unity for higher energy beams. This gives a relative uncertainty for the displacement factor which is at least 100 times larger than the relative uncertainty of the ionization ratio $R$. For example, if we assume the experimental uncertainty on $R$ in the ${ }^{60} \mathrm{Co}$ beam is $0.2 \%$, then the uncertainty on DF will be at least $20 \%$. Aside from the fluctuations of the DF values, it is expected that the values should not depend on depth since transient charged particle equilibrium exists in photon beams and the electron fluence spectra change little versus depth.

For a ${ }^{60} \mathrm{Co}$ beam, the measured displacement factor DF is $0.4 \% \mathrm{~mm}^{-1}$ (see figure 2). Based on this result, the IAEA's TRS-398 (2001) uses the following equation to calculate $P_{\mathrm{gr}}$ values in the ${ }^{60} \mathrm{Co}$ beam for cylindrical chambers of inner radius $r_{\text {cyl }}$ :

$$
P_{\mathrm{gr}}=1-\mathrm{DF} \times r_{\mathrm{cyl}},
$$

where DF $=0.004 \mathrm{~mm}^{-1}$. For a Farmer chamber with $r_{\text {cyl }}=3 \mathrm{~mm}$, equation (3) gives a $P_{\mathrm{gr}}$ value of 0.988 in a ${ }^{60} \mathrm{Co}$ beam. Johansson et al (1977) also used equation (3) to calculate $P_{\text {gr }}$ values, though the equation was not explicitly given in the paper. For high-energy linac photon beams, the TRS-398 Code of Practice did not explicitly give values of $P_{\text {repl }}$ (i.e., $p_{\text {dis }}$ for photon beams) but referred to the values given in TG-21 (AAPM 1983), and stated that the differences between the measured values by Johansson et al and the values in TG-21, which 
Table 1. Comparison of $P_{\text {repl }}$ values for a Farmer chamber ( $3 \mathrm{~mm}$ radius) calculated directly in a water phantom to those obtained from the simulation of Johansson et al's experiments, i.e. by using equations (1) and (5), for different beam qualities. For each beam, the displacement factors, DF, which are calculated from both the normalized and the un-normalized chamber responses, are calculated at three depths $(5,10$ and $15 \mathrm{~cm})$ in a PMMA phantom and the average DF value is used to obtain the $P_{\text {repl }}$ values in the PMMA phantom (1st and 2 nd columns). The number in bracket(s) represents the one standard deviation statistical uncertainty in the last $\operatorname{digit}(\mathrm{s})$.

\begin{tabular}{llll}
\hline & \multicolumn{2}{l}{$P_{\text {repl from simulation of experiments }}$} & \\
\cline { 2 - 3 } & $\begin{array}{l}\text { with } \\
\text { normalization }\end{array}$ & $\begin{array}{l}\text { without } \\
\text { normalization }\end{array}$ & $\begin{array}{l}P_{\text {repl }} \\
\text { calculated in water }\end{array}$ \\
\hline Co & $0.9872(12)$ & $0.9957(12)$ & $0.9961(5)$ \\
$4 \mathrm{MV}$ & $0.9902(11)$ & $0.9955(11)$ & $0.9966(6)$ \\
$6 \mathrm{MV}$ & $0.9921(9)$ & $0.9956(9)$ & $0.9971(4)$ \\
$18 \mathrm{MV}$ & $0.9934(8)$ & $0.9976(8)$ & $0.9971(4)$ \\
\hline
\end{tabular}

can be as large as $0.6 \%$ for a Farmer chamber, are consistent within the estimated uncertainties. However, the $P_{\text {gr }}$ (i.e., $P_{\text {repl }}$ ) values calculated by using equation (3) with $\mathrm{DF}=0.004 \mathrm{~mm}^{-1}$ are significantly different from those given in our previous study (Wang and Rogers 2008a).

As mentioned in the previous section, in order to reproduce the experimental results, we have normalized the calculated depth-ionization curves at $d_{\max }$. However, it turns out this normalization procedure is unreasonable. To investigate this issue further, we need to analyze the measurements more carefully. First, when measuring the phantom dose with two chambers of different radii, $r_{1}$ and $r_{2}$, from the definition of $P_{\text {repl }}$ and since $P_{\text {wall }}$ and the stopping-power ratio are the same for both chambers, the following equation must hold for measurements with the central axis of the chambers at the same depth:

$$
J_{\text {air }, 1} P_{\text {repl }, 1}=J_{\text {air }, 2} P_{\text {repl }, 2},
$$

where $J_{\mathrm{air}, i}$ is the absolute mass ionization reading from chamber $i$ with radius $r_{i}$, and $P_{\mathrm{repl}, i}$ is the replacement correction factor for chamber $i$. Next, we assume for a cylindrical chamber of inner radius $r$, the value of $P_{\text {repl }}$ can be expressed as (Wang and Rogers 2008a, Nahum 1994):

$$
P_{\text {repl }}(r)=1-k r
$$

where $k$ only depends on the radiation beam quality. For two chambers of radii $r_{1}$ and $r_{2}$, if one uses the $P_{\text {repl }}$ values in equation (5), substitutes them in equation (4) and solves for $k$, one obtains

$$
k=\frac{1-\left(J_{\mathrm{air}, 1} / J_{\mathrm{air}, 2}\right)}{r_{2}-\left(J_{\mathrm{air}, 1} / J_{\mathrm{air}, 2}\right) r_{1}} .
$$

Since the ionization ratio $J_{\mathrm{air}, 1} / J_{\mathrm{air}, 2}$ is very close to 1 , it is a good approximation to replace it by 1 in the denominator of equation (6). Then one arrives at equation (1) and $k$ is just the displacement factor. Therefore we have derived equation (1) based on equations (4) and (5). Note that we did not do any normalization in the procedure. This suggests that the normalization at $d_{\max }$ of the measured depth-ionization curves in the experiments, which came from the assumption that the mass ionizations for different chambers are the same at $d_{\max }$, is the cause of the difference between the measured and the Monte Carlo calculated $P_{\text {repl }}$ values. To verify this, we compared the $P_{\text {repl }}$ values for a Farmer chamber calculated directly in a water phantom (see next section) to those obtained from the simulation of Johansson's experiments with the displacement factors calculated from both the normalized (at $d_{\max }$ ) and the un-normalized chamber responses, as shown in table 1 . It is seen that the $P_{\text {repl }}$ value for the 


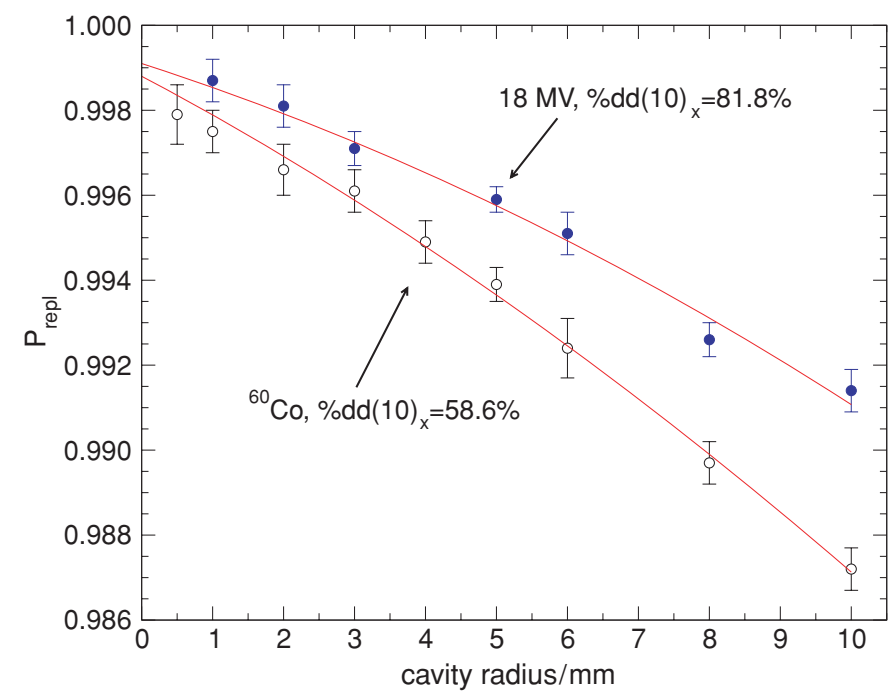

Figure 3. The replacement correction factors for cylindrical chambers $(2 \mathrm{~cm}$ length $)$ at a depth of $10 \mathrm{~cm}$ in a water phantom as a function of chamber radius in a ${ }^{60} \mathrm{Co}$ beam and in an $18 \mathrm{MV}$ linac beam, both of which have a $100 \mathrm{~cm} \mathrm{SSD}$ and a $10 \times 10 \mathrm{~cm}^{2}$ field size. The solid lines are the values calculated by using equation (7).

${ }^{60} \mathrm{Co}$ beam calculated from the normalized chamber response $(0.987)$ is very close to the value calculated by using equation (3) above (0.988), that is, our simulation results 'agree' with the measurements if the chamber responses are normalized at $d_{\max }$. However, if the chamber responses are not normalized, the simulation results agree with $P_{\text {repl }}$ values calculated directly using the Monte Carlo method in a water phantom (Wang and Rogers 2008a). Although the experiments were done in a PMMA phantom, the difference between $P_{\text {repl }}$ values in water and in PMMA for a Farmer chamber is less than $0.1 \%$.

Figure 1 shows a $0.5 \%$ difference in the maximum mass ionization at $d_{\max }$ between the $3 \mathrm{~mm}$ chamber and the $7 \mathrm{~mm}$ chamber. The $d_{\max }$ value for these two chambers is also different: one at $0.50 \mathrm{~cm}$ and the other at $0.67 \mathrm{~cm}$. The $P_{\text {repl }}$ values for the $3 \mathrm{~mm}$ and $7 \mathrm{~mm}$ chambers at the corresponding $d_{\max }$ can be estimated from the figure as the ratio between the depth-ionization value and the cavity-ionization data. This is actually the 'SPR method' for calculating the values of $P_{\text {repl }}$ (Wang and Rogers 2008a). The values thus found are 0.998 and 0.996 , respectively, and they are very close to the directly calculated $P_{\text {repl }}$ values for the cylindrical cavity of the same size at $10 \mathrm{~cm}$ depth in a water phantom (see figure 3 ). In figure 1 , the ionization value on the depth-ionization curve decreases by about $0.7 \%$ from $0.50 \mathrm{~cm}$ depth to $0.67 \mathrm{~cm}$ depth. This accounts for the $0.5 \%$ difference in the mass ionizations at $d_{\max }$ between the $3 \mathrm{~mm}$ chamber and the $7 \mathrm{~mm}$ chamber. The residual $0.2 \%$ is the difference in $P_{\text {repl }}$ values for the two chambers. Thus the $0.5 \%$ difference in the maximum mass ionizations between the $3 \mathrm{~mm}$ and the $7 \mathrm{~mm}$ chambers comes from the attenuation of the depth-dose curve.

The results given above demonstrate that the normalization procedure for the depthionization curves measured by Johansson et al is not correct and it gives incorrect results in determining the displacement factors. Thus the interpretation of the measured values as $P_{\text {repl }}\left(p_{\text {dis }}\right)$ for cylindrical chambers in photon beams is not correct in IAEA's TRS-398 Code of Practice, just as the values in TG-21 based on Cunningham and Sontag's values were 


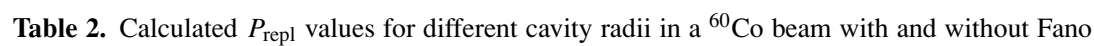
conditions (i.e., no attenuation, no scatter). Calculations are done with the LDW method (Wang and Rogers 2008a) for cylindrical cavities at a depth of $10 \mathrm{~cm}$ in a water phantom. The number in brackets represents the one standard deviation statistical uncertainty in the last digit.

\begin{tabular}{llll}
\hline Cavity radius & $0.5 \mathrm{~mm}$ & $3 \mathrm{~mm}$ & $5 \mathrm{~mm}$ \\
\hline$P_{\text {repl }}$ (normal) & $0.9979(7)$ & $0.9961(5)$ & $0.9939(4)$ \\
$P_{\text {repl }}$ (Fano) & $0.9991(7)$ & $0.9993(6)$ & $0.9997(6)$ \\
\hline
\end{tabular}

based on an incorrect interpretation (Wang and Rogers 2008a). It should be mentioned that Johansson et al also determined $P_{\text {repl }}\left(p_{\text {dis }}\right)$ in a different approach by comparing depthionization curves measured by cylindrical chambers to that by a plane-parallel chamber whose front wall was used as the point of measurement. This resulted in an offset of the effective point of measurement (or radial displacement as called by Johansson et al) ranging from $0.5 r$ to $0.9 r$ (Johansson et al 1977) which was the basis of the $0.6 r$ offset used by current dosimetry protocols for measuring depth-dose curves in photon beams. It is likely that these depth-ionization curves were also normalized at $d_{\max }$ when making the comparisons, so it is susceptible to the same problem. In addition, we have shown that the effective point of measurement for plane-parallel chambers in photon beams is at the cavity center instead of the inner face of the front wall (Wang and Rogers 2008a, 2008b ). This means that the offsets of the effective point of measurement are smaller than that measured using this comparison technique. In other words, the $P_{\text {repl }}$ values should be larger, closer to unity.

\section{3. $P_{\text {repl }}$ calculation results and discussion}

In our previous study (Wang and Rogers 2008a), the values of $P_{\text {repl }}$ at a depth of $5 \mathrm{~cm}$ in a water phantom for a ${ }^{60} \mathrm{Co}$ beam at $80 \mathrm{~cm}$ SSD were calculated for cylindrical chambers of length $2 \mathrm{~cm}$ with various radii and for cylindrical chambers of cavity diameter $6 \mathrm{~mm}$ with a length from $2 \mathrm{~cm}$ to $0.5 \mathrm{~cm}$. The $P_{\text {repl }}$ value for a $6 \mathrm{~mm}$ diameter chamber was found to be $0.9974 \pm 0.07 \%$; and the value only varies by $0.2 \%$ for the chamber length from $2 \mathrm{~cm}$ to $0.5 \mathrm{~cm}$. The present study uses the LDW (low-density water) method (Wang and Rogers 2008a) in which the air cavity is replaced by a low-density water material with all the characteristics of water except that its density is the same as that of air. The systematic uncertainty of using the LDW method to calculate the $P_{\text {repl }}$ values for chambers in photon beams has been analyzed recently by Wang et al (2009) with the conclusion that the systematic uncertainty is at most $0.2 \%$. The cavity radius variation of $P_{\text {repl }}$ values is calculated for cylindrical thimble chambers of length $2 \mathrm{~cm}$ at a depth of $10 \mathrm{~cm}$ in a water phantom irradiated in both a ${ }^{60} \mathrm{Co}$ beam and an $18 \mathrm{MV}$ photon beam at $100 \mathrm{~cm} \mathrm{SSD}$, with $10 \times 10 \mathrm{~cm}^{2}$ field size. The beam quality dependences of $P_{\text {repl }}$ for a Farmer chamber (radius $3 \mathrm{~mm}$ ) and for a larger cylindrical chamber (radius $5 \mathrm{~mm}$ ) are also calculated for photon beams of energy ranging from ${ }^{60} \mathrm{Co}$ to $18 \mathrm{MV}$, all at $100 \mathrm{~cm}$ SSD and at a depth of $10 \mathrm{~cm}$. Fano's theorem tells us that in a uniform field the electron fluence spectrum remains unchanged irrespective of the material density. In other words, under Fano conditions, the value of $P_{\text {repl }}$ calculated by the LDW method should be unity. To verify this, we calculated the $P_{\text {repl }}$ values for cavities of $0.5,3$ and $5 \mathrm{~mm}$ radii at $10 \mathrm{~cm}$ depth in a ${ }^{60} \mathrm{Co}$ beam of $10 \times 10 \mathrm{~cm}^{2}$ at $100 \mathrm{~cm}$ SSD under Fano conditions, i.e. with primary photons regenerated after each interaction and scattered photons being discarded, and the results are tabulated in table 2 . As expected, $P_{\text {repl }}$ values are very close to unity under Fano conditions. 

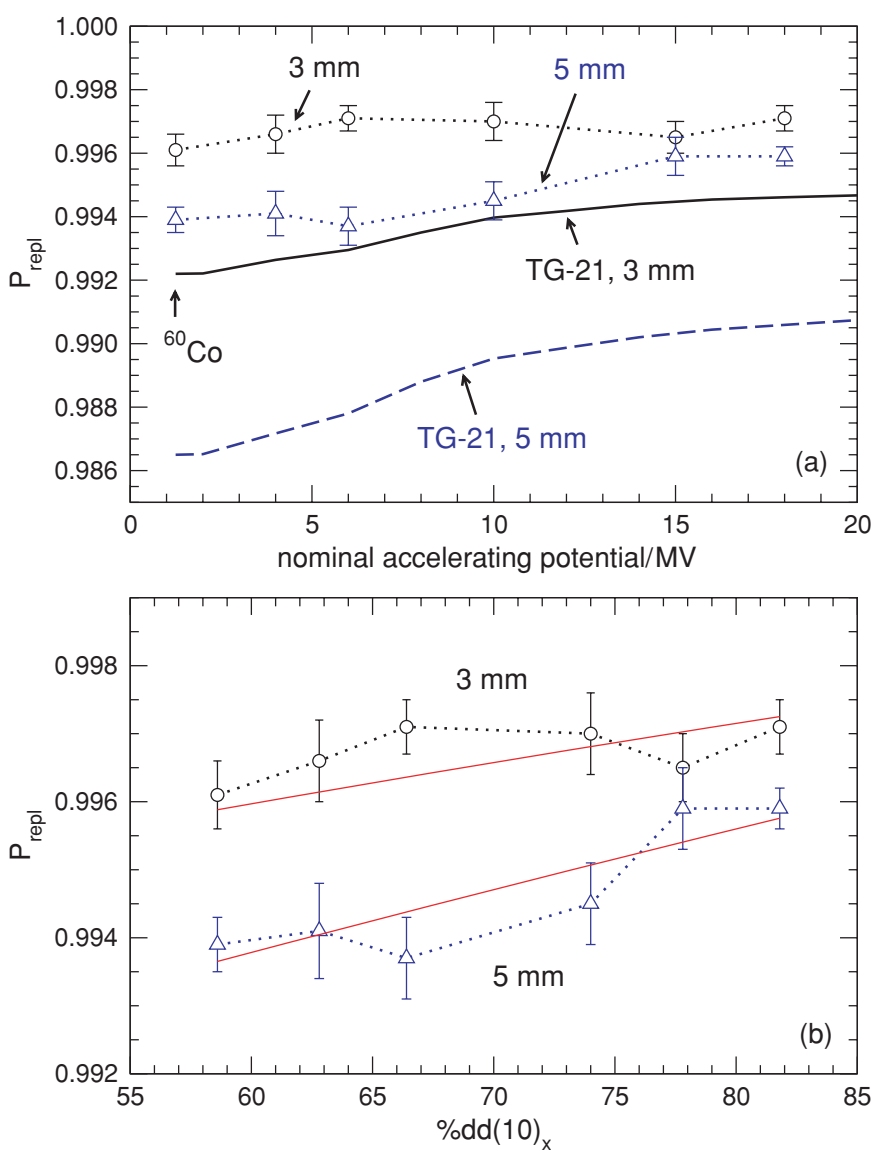

Figure 4. The calculated beam quality dependence of the replacement correction factor for a Farmer chamber ( $3 \mathrm{~mm}$ radius) and a larger cylindrical chamber ( $5 \mathrm{~mm}$ radius) in photon beams. In panel (a), nominal accelerating potential is used as a beam quality specifier so as to compare the values in TG-21. In panel (b), the photon component of the percent depth dose at $10 \mathrm{~cm}$ $\left(\% \mathrm{dd}(10)_{x}\right)$ is the beam quality specifier. The solid lines in panel (b) are the values calculated by using equation (7).

The calculated radial dependence of $P_{\text {repl }}$ values for the ${ }^{60} \mathrm{Co}$ beam and the $18 \mathrm{MV}$ photon beams are shown in figure 3. The solid lines in panel (b) are the fitted curves for the data points (see later). As expected from equation (5), there is a linear relation between the value of $P_{\text {repl }}$ and the cavity radius $r$, with the possible exception of very small radius $(<1 \mathrm{~mm})$. It is expected that $P_{\text {repl }}=1$ for $r=0$, although the fitted curves give values of 0.9988 and 0.9991 for the ${ }^{60} \mathrm{Co}$ beam and the $18 \mathrm{MV}$ linac beam, respectively. However this is only a $0.1 \%$ difference which is about the level of uncertainty of the EGSnrc code system in calculating ion chamber responses (Kawrakow 2000b) or about the systematic uncertainty in calculating $P_{\text {repl }}$ values (Wang et al 2009). The results for the beam quality dependence of the $P_{\text {repl }}$ values are shown in figure 4 and compared to the TG-21 values (which are used in TG-51). It is seen that there is essentially no beam quality dependence of the calculated $P_{\text {repl }}$ values for the Farmer chamber; and it is notable that there is a significant difference between the calculated $P_{\text {repl }}$ values and the TG-21 values which came from Cunningham and Sontag's experiment. For a Farmer chamber in a ${ }^{60} \mathrm{Co}$ beam, the TG- 21 value is $0.4 \%$ lower than it should be. Practically, this means 


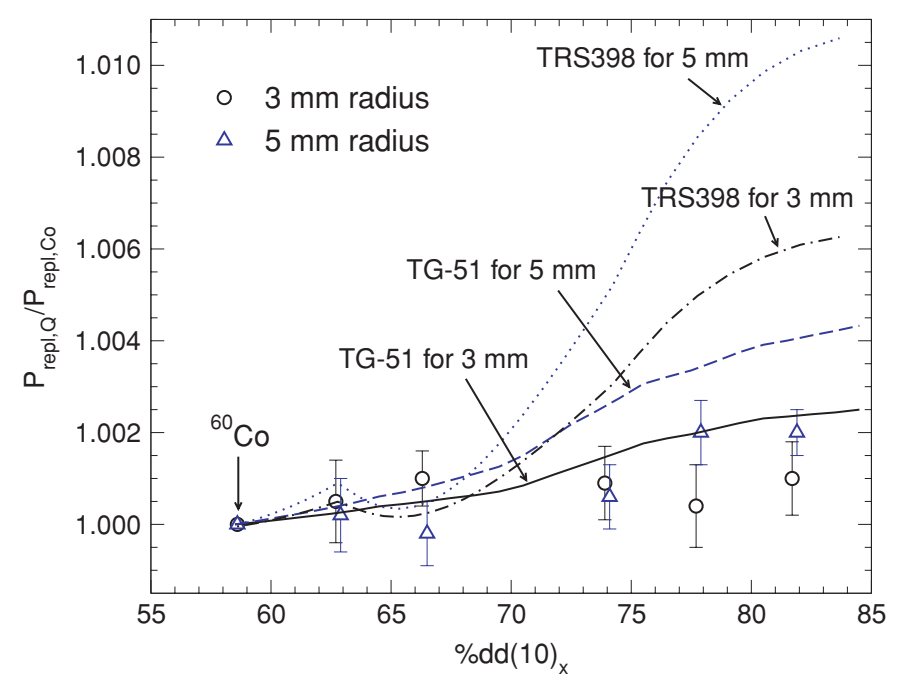

Figure 5. The beam quality dependence of the ratio of $P_{\text {repl }}$ values for a beam of quality $Q$ to that of ${ }^{60} \mathrm{Co}$ for both a Farmer-type chamber $(3 \mathrm{~mm}$ radius) and a larger cylindrical chamber ( $5 \mathrm{~mm}$ radius). The symbols are calculated in this study and the lines are the values used in either TG-51(TG-21) or IAEA's TRS-398.

that a patient treated in the ${ }^{60} \mathrm{Co}$ beam calibrated with TG-21 using a Farmer chamber would be overdosed by $0.4 \%$ due to the incorrect $P_{\text {repl }}$ value. For a high-energy photon beam (e.g., $18 \mathrm{MV}$ ), the discrepancy is still $0.2-0.3 \%$. The difference is even larger for a larger chamber: as shown in figure 4(a), the calculated $P_{\text {repl }}$ values for the $5 \mathrm{~mm}$ radius chamber are at least $0.5 \%$ higher than the TG-21 values for all beam qualities. Since TG-21 used the individual $P_{\text {repl }}$ values directly, it is more susceptible to the changes of these values. On the other hand, one major benefit of the TG-51 dosimetry protocol (also the IAEA TRS-398 Code of Practice) is that only the ratios of various correction factors are involved. Figure 5 shows the ratio of the value of $P_{\text {repl }}$ for a beam of quality $Q$ to that for a ${ }^{60} \mathrm{Co}$ beam as a function of the beam quality $\% \mathrm{dd}(10)_{x}$. For a ${ }^{60} \mathrm{Co}$ beam calibrated with TG-51, the calibration result is always the same whichever value of $P_{\text {repl }}$ is used. For both the Farmer chamber ( $3 \mathrm{~mm}$ radius) and the $5 \mathrm{~mm}$ radius chamber, the discrepancy between the ratio in this study and that used in TG-51 is at most $0.2 \%$ and this largest discrepancy is only for high-energy linac beams; in contrast in TG-21, as we have seen, the difference of the $P_{\text {repl }}$ values for the $5 \mathrm{~mm}$ radius chamber is at least $0.5 \%$ for all beam qualities. Also shown in figure 5 are the values of the ratios used in the IAEA TRS-398 Code of Practice, based on the $P_{\text {repl }}$ values used in TRS-398 (Andreo, private communication, 2008). For the Farmer chamber, the discrepancy between the ratio in this study and that used in TRS-398 can be as large as 0.5\%; and it is even larger for a larger chamber. The original photon beam quality specifier for the TRS-398 data is $T P R_{10}^{20}$, and for this figure it has been converted to \% dd(10) $x$ by an empirical formula introduced for 'clinic-like' beams by Kalach and Rogers (2003).

Using the data in figures 3 and 4(b), one may derive an empirical formula for the values of $P_{\text {repl }}$ for $2 \mathrm{~cm}$ long cylindrical chambers in photon beams as (with $0.1 \%$ uncertainty):

$$
\begin{aligned}
P_{\text {repl }}=0.9974 & -0.00183 r+3.36 \times 10^{-5} \% d d(10)_{x}-2.7 \times 10^{-5} r^{2} \\
& -1.6 \times 10^{-7}\left(\% d d(10)_{x}\right)^{2}+1.58 \times 10^{-5} r \% d d(10)_{x},
\end{aligned}
$$


where $r$ is the cavity radius of the chamber in $\mathrm{mm}$, and $\% d d(10)_{x}$ is the photon beam quality specifier, i.e. percent depth dose at $10 \mathrm{~cm}$ depth, excluding electron contamination. The values of $P_{\text {repl }}$ calculated from equation (7) are shown in figures 3 and $4(\mathrm{~b})$ as solid lines. If $T P R_{10}^{20}$ is used as the photon beam quality specifier, a similar equation for heavily filtered 'clinic-like' beams is

$$
\begin{aligned}
P_{\text {repl }}=1.0021 & -0.00188 r-0.0108 T P R_{10}^{20}-2.5 \times 10^{-5} r^{2} \\
& +0.009\left(T P R_{10}^{20}\right)^{2}+0.00169 r T P R_{10}^{20},
\end{aligned}
$$

where the values of $T P R_{10}^{20}$ are obtained from \% $\mathrm{dd}(10)_{x}$ by the empirical formula of Kalach and Rogers (2003). Equations (7) and (8) are valid for cavities with a length of $2 \mathrm{~cm}$, radii from $1 \mathrm{~mm}$ to $10 \mathrm{~mm}$, and for \% $\mathrm{dd}(10)_{x}\left(T P R_{10}^{20}\right)$ values from $58 \%(0.57)$ to $82 \%(0.80)$.

\section{Conclusions}

EGSnrc Monte Carlo simulation codes are used to simulate Johansson's experimental procedures for determining the values of the replacement correction factors (or displacement factors in the IAEA's terminology) for cylindrical chambers in high-energy photon beams. The simulation results agree well with the measurements if the chamber responses versus depth are normalized at $d_{\max }$ as done in the original experiments, but the values obtained this way differ from the values calculated by the direct Monte Carlo methods of calculating the replacement correction factors. Johansson et al normalized their depth-ionization curves at $d_{\max }$ because they assumed that the chambers of different radii gave the same maximum mass ionization at the respective $d_{\max }$, which turns out not to be correct according to our results. On the other hand, if the chamber responses are not normalized at $d_{\max }$, then the simulation results agree with the $P_{\text {repl }}$ values calculated by the direct Monte Carlo methods. This demonstrates that the normalization procedure used in the experiments is incorrect as it is based on an incorrect assumption, and thus the interpretation of Johansson's experimental values as $P_{\text {repl }}$ in the IAEA TRS-398 Code of Practice is not correct. The values of the replacement correction factors for cylindrical chambers of different radii in various high-energy photon beams are calculated and an empirical formula is given. For Farmer chambers, there is essentially no beam quality dependence for the replacement correction factors in high-energy photon beams. In comparison with the values of the replacement correction factors used in the TG-21 and TG-51 dosimetry protocols, it is found that the differences are more significant for the TG21 dosimetry protocol and may lead to radiation source calibration errors of up to $0.4 \%$. However, in the TG-51 dosimetry protocol, since only the ratio of the correction factors is involved, the difference in the replacement correction factors can only lead to a radiation source calibration result differing by at most $0.2 \%$. These results demonstrate the robustness of the TG-51 dosimetry protocol. For the IAEA TRS-398 Code of Practice, the difference in the replacement correction factors can lead to a calibration deviation of more than $0.5 \%$ for Farmer-like chambers in high-energy photon beams.

\section{Acknowledgments}

The authors are grateful to Dr Karl-Axel Johansson for detailed information on determining the displacement factors and to the referees for perceptive comments. We also thank Pedro Andreo for providing us information and a routine related to the calculation of the displacement correction factors in TRS-398. This work is supported by an Ontario Graduate Scholarship (OGS), NSERC, the Canada Research Chairs program, CFI and OIT. 


\section{References}

AAPM TG-21 1983 A protocol for the determination of absorbed dose from high-energy photon and electron beams Med. Phys. 10 741-71

Almond P R, Biggs P J, Coursey B M, Hanson W F, Huq M S, Nath R and Rogers D W O 1999 AAPM's TG-51 protocol for clinical reference dosimetry of high-energy photon and electron beams Med. Phys. 26 1847-70

Andreo P, Linborg L and Medin J 1991 On the calibration of plane-parallel ionisation chambers using ${ }^{60} \mathrm{Co}$ beams Med. Phys. 18 326-7

Andreo P 2008 private communication

Cunningham J R and Sontag M R 1980 Displacement corrections used in absorbed dose determinations Med. Phys. 7 672-6

Halbleib J 1988 Structure and operation of the ITS code system Monte Carlo Transport of Electrons and Photons ed T M Jenkins, W R Nelson, A Rindi, A E Nahum and D W O Rogers (New York: Plenum) pp 249-62

Absorbed Dose Determination in External Beam Radiotherapy: An International Code of Practice for Dosimetry Based on Standards of Absorbed Dose to Water (Technical Report Series vol 398) (Vienna: IAEA)

Johansson K A, Mattsson L O, Lindborg L and Svensson H 1977 Absorbed-dose determination with ionization chambers in electron and photon beams having energies between 1 and $50 \mathrm{MeV}$ IAEA Symposium Proceedings, IAEA-SM-222/35 IAEA, Vienna pp 243-70

Kalach N I and Rogers D W O 2003 Which accelerator photon beams are "clinic-like" for reference dosimetry purposes? Med. Phys. 30 1546-55

Kawrakow I 2000a Accurate condensed history Monte Carlo simulation of electron transport. I. EGSnrc, the new EGS4 version Med. Phys. 27 485-98

Kawrakow I 2000b Accurate condensed history Monte Carlo simulation of electron transport. II. Application to ion chamber response simulations Med. Phys. 27 499-513

Kawrakow I 2005 EGSnrc C++ class library Technical Report PIRS-898, National Research Council of Canada, Ottawa, Canada. http://www.irs.inms.nrc.ca/EGSnrc/pirs898/

Kawrakow I and Rogers D W O 2000 The EGSnrc Code System: Monte Carlo simulation of electron and photon transport Technical Report PIRS-701 (Ottawa, Canada: National Research Council of Canada)

Mora G, Maio A and Rogers D W O 1999 Monte Carlo simulation of a typical ${ }^{60}$ Co therapy source Med. Phys. 26 2494-502

Nahum A E 1994 Perturbation Effects in Dosimetry Technical Report ICR-PHYS-1/94, Joint Dept of Physics, The Royal Marsden Hospital, Sutton, Surrey, SM2 5PT, UK

Rogers D W O 1996 Fundamentals of dosimetry based on absorbed-dose standards Teletherapy Physics, Present and Future ed J R Palta and T R Mackie (Washington, DC: AAPM) pp 319-56

Sheikh-Bagheri D and Rogers D W O 2002 Monte Carlo calculation of nine megavoltage photon beam spectra using the BEAM code Med. Phys. 29 391-402

Wang L L W, La Russa D J and Rogers D W O 2009 Systematic uncertainties in the Monte Carlo calculation of ion chamber replacement correction factors Med. Phys. 36 (submitted)

Wang L L W and Rogers D W O 2008a Calculation of the replacement correction factors for ion chambers in megavoltage beams by Monte Carlo simulation Med. Phys. 35 1747-55

Wang L L W and Rogers D W O 2008b The replacement correction factor for the BIPM flat cavity ion chamber and the value of W/e Med. Phys. 35 4410-6 